\title{
Solar neutrino results from Super-Kamiokande
}

\author{
Yusuke Koshio*广 \\ Kamioka observatory, ICRR, Univ. of Tokyo \\ E-mail: koshio@icrr.u-tokyo.ac.jp
}

Recent results of the Super-Kamiokande (SK) solar neutrino measurements are presented. The main goal of SK's solar analysis is to observe the MSW effect, i.e. a solar neutrino energy spectrum distortion induced by matter in the Sun, and a day/night solar neutrino flux asymmetry induced by matter in the Earth. Thanks to the improvement of the detector's water circulation system, low energy background levels have been reduced and clear solar neutrino signals are seen for 4.0-4.5 MeV electron kinetic energies, along with some indications of a signal even down to 3.5-4.0 MeV. The combined energy spectrum and day/night solar neutrino flux from period I to period IV in SK will be presented. A global oscillation analysis has been carried out using SK-I, II, III, and IV data, and by combining these results with the results of other solar neutrino experiments as well as the KamLAND reactor neutrino experiment. The results of this global analysis will also be presented.

36th International Conference on High Energy Physics,

July 4-11, 2012

Melbourne, Australia

\footnotetext{
*Speaker.

${ }^{\dagger}$ Super-Kamiokande collaboration
} 


\section{Introduction}

The origin of the energy in the Sun is the following nuclear fusion reaction which generates two neutrinos,

$$
4 p \rightarrow \alpha+2 e^{+}+2 v_{e}
$$

Neutrinos, generated in the Sun's core, reach the earth $\sim 8$ minutes later, unlike other particles. Therefore, solar neutrino measurements directly carry information about the current status of the center of the Sun.

Over the past decade, a solar neutrino oscillation scenario has been established by the solar neutrino experiments (SK, SNO, and radiochemical experiments) and the KamLAND reactor experiment. The next step then for solar neutrino measurements is the precise determination of the oscillation parameters and the role of matter therein. SK plays an important role by collecting high statistics solar neutrino data to attempt measurements of a day-night flux difference and a distortion of the energy spectrum.

SK is a 50000 ton imaging water Cherenkov detector, and it is located $1000 \mathrm{~m}$ underground (2700 m of water equivalent) in the Kamioka mine in Gifu Prefecture, Japan, to shield against cosmic ray muons. Cherenkov light generated by charged particles scattered by neutrinos in water are detected by 1114620 -inch photomultiplier tubes. The experiment started normal data taking on April 1996, by March 2012 we have accumulated 3904 days of solar neutrino data. SK detects solar neutrinos through neutrino-electron elastic scattering, $v+e \rightarrow v+e$, where the energy, direction, and time of the recoil electron are measured. Due to its large (22.5 kiloton) fiducial mass, SK makes precise measurements of ${ }^{8} \mathrm{~B}$ solar neutrinos, including precision information on their energy spectrum and its time variation.

\section{Recent results}

In this paper, we first report solar neutrino results from the SK-IV period, which started data taking following new electronics installation in September 2008. By the end of March 2012, 1069.3 days of SK-IV solar neutrino data suitable for analysis had been taken.

The most important recent progress for solar neutrino analysis is that the background rate in the lower energy region (below $6 \mathrm{MeV}$ ) has been reduced by a factor of more than three compared to the SK-I period (the first 5 years). It comes from many efforts we have made to improve the SK water system and to increase the precision of the detector calibrations. It has allowed a lower analysis energy threshold: down to $4.0 \mathrm{MeV}$ from $4.5 \mathrm{MeV}$ of kinetic energy. A clear solar neutrino signal in the 3.5-4.0 MeV energy region can also be seen at more than $7 \sigma$.

\subsection{Flux}

The total flux systematic error during SK-IV is $\pm 1.7 \%$, which is nearly half that of the SK-I period, ${ }_{-3.2}^{+3.5} \%$ [1]. This reduction comes from improvements in the uncertainty of the fiducial volume size ( $\pm 1.3 \%$ in SK-I, $\pm 0.17 \%$ in SK-IV), better understanding of the absolute energy scale ( $\pm 0.64 \%$ in SK-I, $\pm 0.54 \%$ in SK-IV), and careful studies of the data reduction. During SK-IV the measured ${ }^{8} \mathrm{~B}$ flux is $\left(2.34 \pm 0.03\right.$ (stat.) \pm 0.04 (sys.) $\left.\times 10^{6} \mathrm{~cm}^{-2} \mathrm{~s}^{-1}\right)$, which is consistent with previous measurements from SK-I, II, and III and is shown in Fig. 1. 


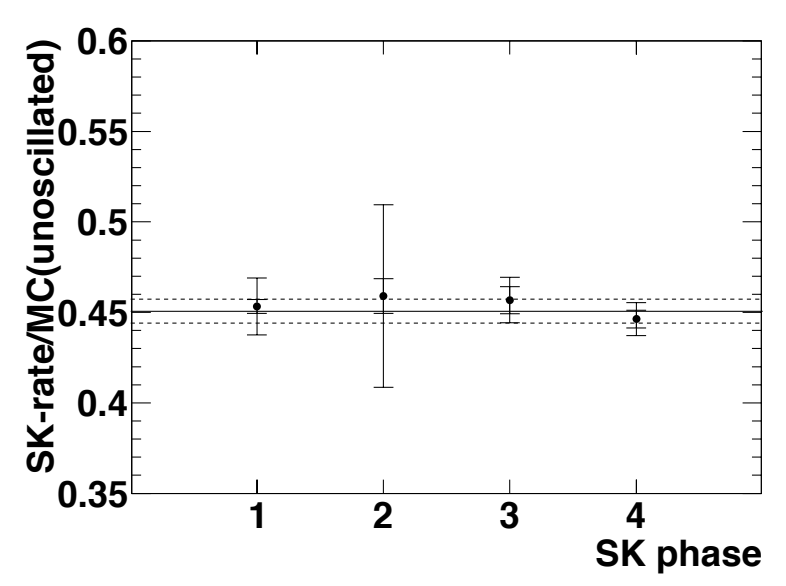

Figure 1: Solar neutrino flux measurements from SK-I to SK-IV with statistical and systematic errors, normalized to the unoscillated MC prediction using a ${ }^{8} \mathrm{~B}$ flux of $5.25 \times 10^{6} / \mathrm{cm}^{2} / \mathrm{sec}$. The dashed lines show the average over all periods. The reason why SK-II has a large uncertainty is half number of PMTs and less sensitivity comparing to other periods.

\subsection{Energy spectrum}

The energy spectrum of the recoil electron is shown in Fig. 2 (right). The vertical axis shows the ratio of the observed energy spectrum to the expectation from the unoscillated MC simulation assuming a ${ }^{8}$ B flux of $5.25 \times 10^{6} / \mathrm{cm}^{2} / \mathrm{sec}$. Fig. 2(left) shows SK-I to SK-IV combined energy spectrum with expectations from global solar and solar+KamLAND as well as flat reduction of the neutrino spectrum. The combined energy spectrum is consistent with the flat prediction, but the level of favoring flat over the upturn is $1.1 \sim 1.9 \sigma$.

\subsection{Day/Night flux differences}

Concerning differences in the day and night fluxes the expected flux asymmetry, defined as $A_{D N}=($ day $-n i g h t) / \frac{1}{2}($ day + night $)$, is about $2 \%$ based on current understanding of neutrino oscillation parameters. Although this is not a large effect, long term observations by SK enable discussion of a finite value of the day-night asymmetry. In our data analysis, a more sophisticated method used in searching for the day/night asymmetry employs a maximum likelihood fit to the amplitude of the expected variation to the entire data sample. The overall amplitude using the combined SK periods is $-2.8 \pm 1.1 \pm 0.5 \%$, which is a $2.3 \sigma$ difference from zero.

The day-night asymmetry is obtained by fitting the observed time variation during the night with its expected variation, meaning that $A_{D N}$ depends on the assumed value of $\Delta m^{2}$. Fig. 3 shows $A_{D N}$ as a function of $\Delta m^{2}$ together with the expectation. The observed $A_{D N}$ is consistent with the expectation using the best fit $\Delta m^{2}$ from both KamLAND and the global solar analysis.

\section{Neutrino oscillation analysis}

A global solar neutrino oscillation analysis has been performed including all SK data (SK-I [1], SK-II [2], SK-III [3], and SK-IV) as well as the most recent results from SNO [4], the radiochemical 

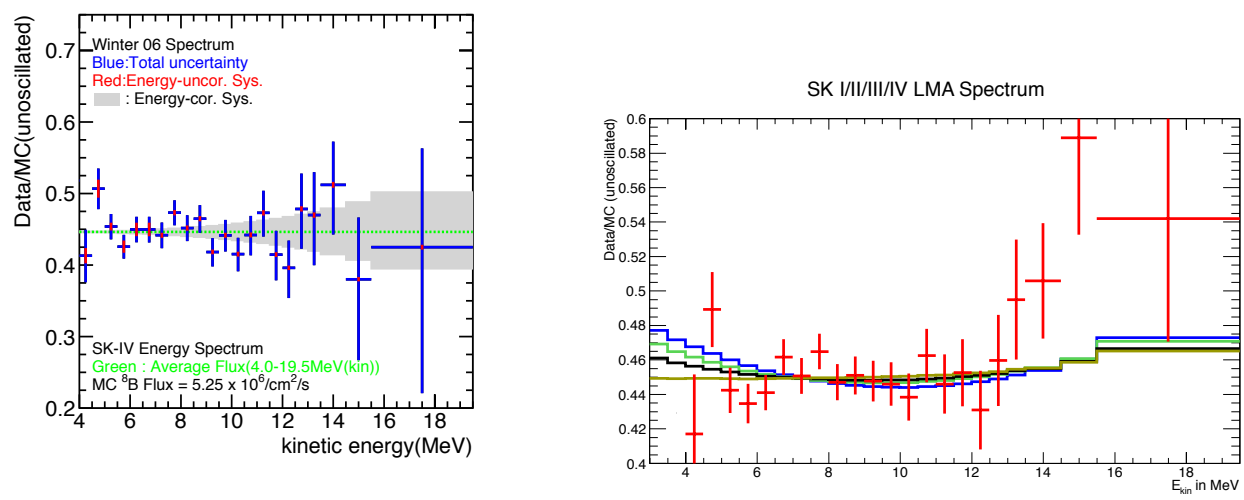

Figure 2: The left figure shows the solar neutrino energy spectrum in SK-IV. Each point shows the ratio of the data to the expected flux using an unoscillated ${ }^{8} \mathrm{~B}$ solar neutrino spectrum. The right shows the spectrum combining SK-I to SK-IV with predictions for (1) $\sin ^{2} \theta_{12}=0.304$ and $\Delta m_{21}^{2}=7.4 \times 10^{-5} \mathrm{eV}^{2}(\mathrm{blue})$, (2) $\sin ^{2} \theta_{12}=0.314$ and $\Delta m_{21}^{2}=4.8 \times 10^{-5} \mathrm{eV}^{2}$ (light blue), (3) flat probability with the best fit of the energy correlated systematic error (black), and (4) flat probability and $d \sigma / d E$ shape for pure $v_{e}+e$ scattering (blond).

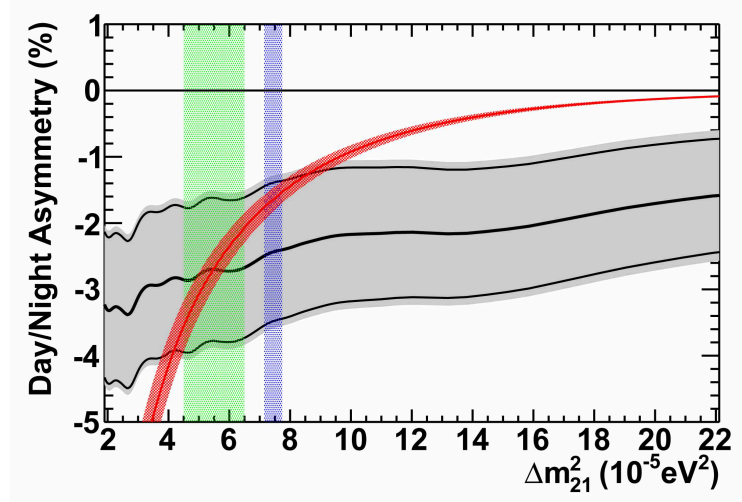

Figure 3: The day-night asymmetry as a function of $\Delta m^{2}$. A thick black line shows the result of the SK fit and the surrounding gray band indicates statistical and systematic uncertainty (thin black lines for statistical error only). The red curve shows the expectation assuming $\sin ^{2}\left(\theta_{12}\right)=0.314$. The light blue and light green vertical bands show the allowed range of $\Delta m^{2}$ from the KamLAND reactor and solar global, respectively.

experiments [5], and the latest ${ }^{7} \mathrm{Be}$ flux measurement from Borexino [6], This analysis was then compared and combined with the reactor neutrino results from KamLAND [7].

Figure 4 shows the allowed region of the neutrino oscillation parameters $\sin ^{2} \theta_{12}$ and $\sin ^{2} \theta_{13}$. The purple contours is combined with global solar neutrino oscillation and KamLAND result. The constraint on $\sin ^{2} \theta_{13}$ was $0.030_{-0.015}^{+0.017}$, which is a roughly $2 \sigma$ hint that $\theta_{13}$ is different from zero. This hint has been discussed prior to 2010 [8] [3]. After 2011, the T2K, Double Chooz, Daya Bay, and Reno experiments presented an evidence for a finite $\theta_{13}$. The combination of their 


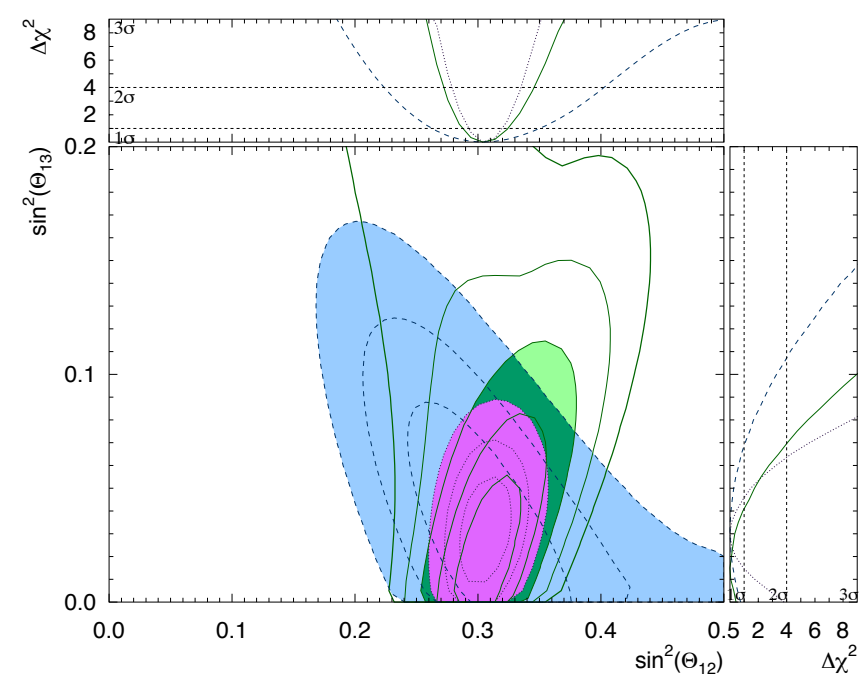

Figure 4: Allowed regions of the neutrino oscillation parameters $\sin ^{2} \theta_{12}$ and $\sin ^{2} \theta_{13}$ from the global solar neutrino analysis (green) and the KamLAND reactor neutrino data (light blue). The purple region shows the contour from the combined global solar and KamLAND reactor analysis. Curves are drawn for each 1- $\sigma$ step between 1 and $5 \sigma$ for the global solar analysis, and 1-3 $\sigma$ for the KamLAND and solar+KamLAND regions. Contours at $3 \sigma$ are filled with their colors.

measurements yields $\sin ^{2} \theta_{13}=0.025_{-0.004}^{+0.003}$ and the result of the combined analysis of the global solar and KamLAND reactor data is consistent with this value.

Figure 5 shows the allowed region of neutrino oscillation parameters in the $\Delta m_{21}^{2}$ and $\sin ^{2} \theta_{12}$ plane assuming $\sin ^{2} \theta_{13}$ is fixed at 0.025 . The obtained parameters from the global solar analysis are $\Delta m_{21}^{2}=\left(4.86_{-0.52}^{+1.44}\right) \times 10^{-5} \mathrm{eV}^{2}$ and $\sin ^{2} \theta_{12}=0.310_{-0.015}^{+0.014}$. Comparing these values with those from KamLAND, $\left(\Delta m_{21}^{2}=\left(7.49_{-0.19}^{+0.20}\right) \times 10^{-5} \mathrm{eV}^{2}\right.$ and $\left.\sin ^{2} \theta_{12}=0.309_{-0.029}^{+0.039}\right)$, there is a $1.8 \sigma$ level tension in the $\Delta m_{21}^{2}$ results, which is evident in the figure. Combining the global solar data with KamLAND, the oscillation parameters become $\Delta m_{21}^{2}=\left(7.44_{-0.19}^{+0.20}\right) \times 10^{-5} \mathrm{eV}^{2}$ and $\sin ^{2} \theta_{12}=$ $0.304 \pm 0.013$.

\section{Summary}

Super-Kamiokande has precisely measured the solar neutrino flux, its time variation, and recoil electron spectrum. A reduction of background and smaller systematic uncertainties has been achieved since SK-IV. As a result of the data analysis covering all periods in SK, no significant energy distortion can be seen, while some hint of non-zero day/night flux asymmetry appears at $2.3 \sigma$ level. In the solar neutrino oscillation analysis combined with KamLAND, oscillation parameters are determined as $\Delta m_{21}^{2}=7.44_{-0.19}^{+0.20} \times 10^{-5} \mathrm{eV}^{2}, \sin ^{2} \theta_{12}=0.304 \pm 0.013$, and $\sin ^{2} \theta_{13}=0.030_{-0.15}^{+0.17}$. For future measurements, the success with which the solar neutrino signal around $3.5 \mathrm{MeV}$ energy can be extracted, currently more than $7 \sigma$, with impact potential discovery of an energy upturn as predicted by the current neutrino oscillation scenario. 


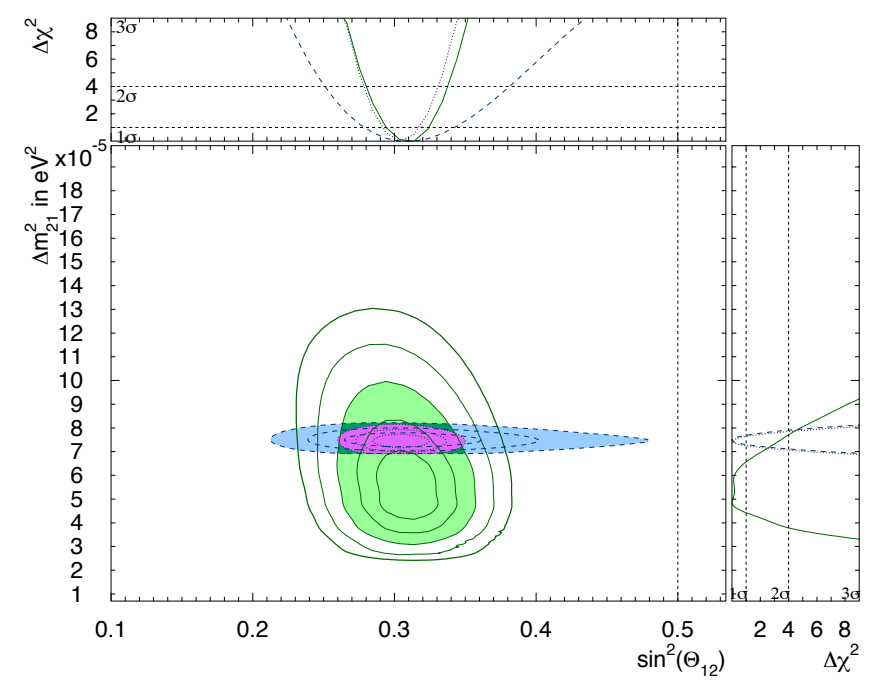

Figure 5: Allowed regions of neutrino oscillation parameters in the $\Delta m_{21}^{2}$ and $\sin ^{2} \theta_{12}$ plane with $\sin ^{2} \theta_{13}$ fixed at 0.025 from the global solar neutrino analysis (green) and the KamLAND reactor neutrino data (light blue). The purple area shows the combined contour of the global solar and the KamLAND reactor analysis. The curves are drawn for each 1- $\sigma$ step from 1-5 $\sigma$ for the global solar, and from 1-3 $\sigma$ for the KamLAND and solar+KamLAND results. Contours at $3 \sigma$ are filled with their colors.

\section{References}

[1] J. Hosaka et al., Solar neutrino measurements in Super-Kamiokande-I, Phys. Rev. D 73 (2006) 112001.

[2] J. P. Cravens et al., Solar neutrino measurements in Super-Kamiokande-II, Phys. Rev. D 78 (2008) 032002 .

[3] K. Abe et al., Solar neutrino results in Super-Kamiokande-III, Phys. Rev. D 83 (2011) 052010.

[4] B. Aharmim et al., Combined Analysis of all Three Phases of Solar Neutrino Data from the Sudbury Neutrino Observatory [nucl-ex/1109.0763].

[5] B. T. Cleveland et al., Measurement of the solar electron neutrino flux with the Homestake chlorine detector Astrophys. J. 496 (1998) 505,

J. N. Abdurashitov et al., Measurement of the solar neutrino capture rate with gallium metal. III. Results for the 2002-2007 data-taking period Phys. Rev. C 80 (2009) 015807.

[6] G. Bellini et al., Precision measurement of the 7Be solar neutrino interaction rate in Borexino Phys. Rev. Lett. 107 (2011) 141302.

[7] A. Gando et al., Constraints on $\theta_{13}$ from a three-flavor oscillation analysis of reactor antineutrinos at KamLAND Phys. Rev. D 83 (2011) 052002.

[8] G. L. Fogli, E. Lisi, A. Marrone, A. Palazzo, and A. M. Rotunno, Evidence of $\theta_{13}>0$ from global neutrino data analysis Phys. Rev. Lett. 101 (2008) 141801. 